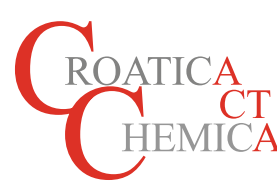

\title{
Auxin Amidohydrolases - From Structure to Function: Revisited
}

\author{
Ana Smolko, ${ }^{1}$ Jutta Ludwig-Müller, ${ }^{2}$ Branka Salopek-Sondi ${ }^{1, *}$
}

\footnotetext{
Department of Molecular Biology, Ruđer Bošković Institute, Bijenička c. 54, 10000 Zagreb, Croatia

2 Institut für Botanik, Technische Universität Dresden, 01062 Dresden, Germany

* Corresponding author's e-mail address: salopek@irb.hr
}

RECEIVED: April 18, 2018 * REVISED: June 4, 2018 औCCEPTED: June 6, 2018

THIS PAPER IS DEDICATED TO DR. BISERKA KoJIĆ-PRODIĆ ON THE OCCASION OF HIS/HER 80 ${ }^{\text {Th }}$ BIRTHDAY

Abstract: The control of plant growth and development is a well-coordinated process between exogenous and endogenous signals. Auxins are plant hormones belonging to the endogenous signals, which control a vast array of different processes. While auxins are growth promoting at low concentrations, higher levels are often inhibitory. Therefore, the tight control of auxin concentrations in a given plant tissue is essential. Among several processes that participate in auxin homeostasis, we focused herein on the process of reversible auxin conjugation that considers the synthesis of inactive auxin conjugates, which can be hydrolyzed back to the active form by so called auxin conjugate hydrolases. Although these proteins have been known for quite some time, their role in plants is still not clear, especially since novel hydrolases with different substrate specificities have been isolated. Thus, we have revisited the knowledge about auxin hydrolases, from their structure and biochemistry to the role in plant development and in dealing with unfavorable climate conditions.

Keywords: auxin, auxin-amidohydrolases, plant development, stress response.

\section{INTRODUCTION}

A UXIN amidohydrolases are metalloenzymes belonging to the amidohydrolase superfamily, the peptidase family M20, subfamily M20D (MEROPS: the Peptidase Database, http://merops.sanger.ac.uk/).[1] Amidohydrolases catalyse a number of reactions including deamination, dephosphorylation, decarboxylation, dechlorination, and isomerization, and are important in amino acid and nucleotide metabolisms as well as biodegradation of agricultural and industrial compounds. ${ }^{[2]}$ They are also one of the important homeostatic regulators of auxins, crucial plant hormones involved in many aspects of plant growth and development, as well as adaptation and survival in extreme environmental conditions. ${ }^{[3,4]}$

High concentrations of free auxins, mostly indole-3acetic acid (IAA), are toxic for plants, so only about $5 \%$ of the total concentration of auxin molecules in plants is in the free (active) form, while the rest is stored in inactive forms, mostly as amino acid and sugar conjugates. ${ }^{[3]}$ Auxin amidohydrolases specifically hydrolyse the amide bond of amino acid conjugated auxins, releasing free active compounds, and contribute in part to auxin homeostasis. Herein, we review knowledge on auxin amidohydrolases: from structure, and biochemistry to their function in plant growth, development and survival in the ever-changing environment.

\section{Auxin: Crucial Plant Hormone}

The research of auxins dates back to Darwin's time. They were the first plant hormones discovered. Auxins are indolic compounds, with an aromatic ring and a carboxylic acid group. ${ }^{[5]}$ Indole-3-acetic acid (IAA) is the most abundant auxin in the plant kingdom, although some other endogenous auxins have been reported, such as indole-3butyric acid (IBA), 4-chloroindole-3-acetic acid (4-Cl-IAA), and phenylacetic acid (PAA) (reviewed by Salopek-Sondi et al.). ${ }^{[6]}$ In order to understand the mechanism of the physiological effects of auxin at the molecular level and to find growth regulators and herbicides suitable for agricultural application, structure-activity correlations for auxins, mostly indole-3-acetic acid (IAA) and a number of its

(c) Br $\mathbf{B r}$ This work is licensed under a Creative Commons Attribution 4.0 International License. 
derivatives, have attracted much attention over the last decades. Thus, structural and physico-chemical parameters, as well as bioactivity of different IAA derivatives such as alkylated and halogenated derivatives of IAA, have been investigated in details. ${ }^{\text {[7-12] }}$

Auxin is involved in an array of diverse responses during the life cycle of a plant, such as cell elongation, cell division, root and shoot growth, apical dominance, flower and fruit development, gravi- and phototropism and many more. ${ }^{[13]}$ While auxin at low levels is actively regulating the above processes, it is inhibitory at higher concentrations. Therefore, it is crucial for proper plant development to control the levels of auxin in a given tissue, e.g. by biosynthesis, transport, degradation or conjugation to amino acids or sugars, which are hydrolyzed upon need for free auxin. ${ }^{[3,14,15]}$ The active IAA overall level in a plant is not only maintained by the amount of IAA synthesized, but also by the quantity of IAA that is released into the "free" state from the conjugated forms. ${ }^{[3,16,17]}$ Over $95 \%$ of the auxin in a plant, depending on the plant tissue and species, ${ }^{[3]}$ can be found in the conjugated form, leaving only a small amount of free hormone available to stimulate and control cellular growth. ${ }^{[17-20]}$ Several groups of auxin conjugates with sugars, amino acids or peptides/proteins have been described so far. ${ }^{[3,15]}$ Whereas in monocots the ester conjugates prevail, in dicots studied to date amide conjugates account for the bulk of conjugated IAA. ${ }^{[3]}$ Overview of amide and ester conjugates present in various plant species has been thoroughly elaborated in reviews by Ludwig-Müller, ${ }^{[3]}$ and Bajguz and Piotrowska. ${ }^{[21]}$ In brief, a few individual IAA amide conjugates have been identified as natural conjugates, for example, IAA-Aspartate (IAA-Asp) and IAAGlutamate (IAA-Glu) have been detected in cucumber ${ }^{[22]}$ and soybean, ${ }^{[23]}$ IAA-Alanine (IAA-Ala) in Picea abies, ${ }^{[24]}$ and IAA-Ala, IAA-Asp, IAA-Leucine (IAA-Leu), and IAA-Glu in Arabidopsis thaliana. ${ }^{[25-27]}$ In Helleborus niger ${ }^{[28]}$ and Arabidopsis ${ }^{[29]}$ a larger number of amide conjugates has recently been identified by the novel approach of immunoaffinity purification fusing labeled standards for IAA amide conjugates, followed by a sensitive liquid chromatography-mass spectrometry (LC-MS). Also, several studies suggested that IAA-peptides and proteins are present in different plant tissues. ${ }^{[30-33]}$

The biosynthesis of amide conjugates is catalyzed by a subset of proteins from the so-called GH3 family. ${ }^{[34,35]}$ The opposite reaction is achieved by releasing the active IAA hormone from amide conjugates by amidohydrolase enzymes that cleave the amide bond between the auxin and the amino acid.

\section{Auxin Amidohydrolases: Discovery}

After the discovery of the first auxin amidohydrolase in Arabidopsis in the year $1995,{ }^{[36]}$ several other IAA amidohydrolases have been isolated from this plant species. ${ }^{[37,20,38]}$ Each of these enzymes has a different, but overlapping substrate specificity. For example, AtILL2 and AtILL1 are $87 \%$ identical, but possess very different substrate specificities. Whereas AtILL2 is highly active with numerous IAA-amide conjugates, AtILL1 is unlikely to contribute to IAA conjugate hydrolysis in vivo due to its low activity. ${ }^{[38]}$ Based on the sequence homology with the $A$. thaliana hydrolases, several members from other plant families were cloned and partially characterized in terms of their expression and some biochemical properties.

Following studies on A. thaliana, another auxin conjugate hydrolase has been isolated and partially characterized from its close relative, Arabidopsis suecica (sILR1). [39] Interestingly, small changes in the amino acid sequence resulted in a different set of auxin conjugates, which were hydrolyzed by the enzyme. ${ }^{[18]}$ (ILR1 preferentially cleaves IAA-Phe and IAA-Leu; ILL1, ILL2 and IAR3 prefer IAA-Ala; sILR1 is more specific for IAA-Gly and IAA-Ala). This result raised interesting evolutionary and physiological questions about the ILR1-like family and how it may have changed in species inside, and in more distant species outside the Brassicaceae family. ${ }^{[40]}$ From the monocot species wheat (Triticum aestivum) an ortholog of the A. thaliana IAR3 was isolated, which showed till then undescribed substrate specificity for longer side chain auxins such as amino acid conjugates IBA and indole-3-propionic acid (IPA). ${ }^{[41]}$ Further characterization of the IAA amino acid conjugate hydrolases from Medicago truncatula and Brassica rapa surprisingly, showed that these hydrolases also preferred IBA and IPA conjugates over IAA conjugates. ${ }^{[41-43]}$

IBA is an auxin derivative used commonly to induce root formation. Although several studies have demonstrated that IBA was found in higher plants as endogenous auxin, ${ }^{[44,45]}$ there are still some questions and controversies about IBA detection and endogenous appearance as well as its role in auxin metabolism. ${ }^{[28]}$ Frick and Strader ${ }^{[46]}$ suggest that IBA to IAA conversion plays roles in plant growth and development that cannot be easily compensated for by other pathways; otherwise, this pathway may be considered as lost, at least in some species. IPA also has some auxin activity, but in addition it has been discussed to have antimicrobial activity. ${ }^{[47]}$ It was identified in several plant species, ${ }^{[48,49]}$ although questions arise about the function IPA has in plants as an endogenous compound. ${ }^{[3]}$ Due to the questionable and still controversial appearance of IBA and IPA as endogenous auxins, the significance of the strong substrate specificities of certain auxin amidohydrolases in vitro toward long chain auxin-amidoconjugates remains unclear.

From the model legume $M$. truncatula a family of auxin conjugate hydrolases was isolated which showed overlapping substrate specificities, ${ }^{[43]}$ thus resembling the 
family of $A$. thaliana hydrolases. Interestingly, in this family of hydrolases, several ones were found which displayed activity towards IAA-Aspartate, ${ }^{[43]}$ earlier described only as a substrate for bacterial IAA conjugate hydrolases. ${ }^{[50]}$

Even though IAR3 enzyme was classified as an IAA amidohydrolase based on its initially examined in vitro activity against IAA conjugates, ${ }^{[51]}$ more recently it was shown it has a dual role participating also in jasmonic acid (JA) homeostasis. ${ }^{[52,53]}$ These results suggest that IAR3/JIH1 from Nicotiana attenuata ${ }^{[52]}$ and Arabidopsis ${ }^{[53]}$ is one of the major players of auxin-jasmonate crosstalk in plant stress responses.

New insights into the auxin amidohydrolases family have been given for the gymnosperm species Sitka spruce (Picea sitchensis) auxin amidohydrolases PsIAR31 and PsIAR32 and Loblolly pine (Pinus taeda) auxin amidohydrolase PtIAR31. ${ }^{[54]}$ Whereas PsIAR31 shows activity towards IAA-Ala, IAA-Isoleucine (IAA-lle), and IAA-glucose (IAAgluc), PsIAR32 recognizes only IAA-Asp and IAA-gluc as substrates. The pine hydrolase PtIAR3 showed activity towards a wider set of conjugates, but it had an 8-fold higher activity towards IBA-Ala than for IAA-Asp, which is interesting considering the appearance of IAA-Asp as a primary naturally occurring auxin conjugate in Scots pine (Pinus sy/vestris). ${ }^{[55]}$ Hence, PtIAR31 was found to be primarily an IBA-conjugate hydrolase, and IAA-Asp was implied in roles other than involvement in catabolic pathways. Also, the redundancy of auxin amidohydrolases in gymnosperms, based on the number of paralogues found in both Sitka spruce and Loblolly pine, indicates redundancy as a common attribute of amidohydrolase protein families, which has developed much earlier in the evolution of higher plants and is not a recent phenomenon seen in angiosperms. ${ }^{[54]}$

\section{Structure and Biochemistry}

Structurally, auxin amidohydrolases are characterized by two perpendicular domains with the larger catalytic domain bearing a binuclear metal center, and the smaller "satellite" domain, usually serves as a dimerization interface and it is proposed to participate in the stabilization and the regulation of enzyme activity (Figure 1.A and 1.B). Auxin amidohydrolases are active in the monomeric form, but, as such a form is highly unstable, the enzymes have a tendency to stabilize by forming polymers in vivo. ${ }^{[42,58]}$ For their activity they require the presence of a reducing agent, such as dithiothreitol, and a metal co-factor. Members of the $M 20$ peptidases generally have two cations in the active site. Bacterial peptidases from the M20 family prefer $\mathrm{Zn}^{2+}$ ions as cofactors, ${ }^{[59]}$ whereas auxin amidohydrolases prefer $\mathrm{Mn}^{2+} \cdot[42,58,60]$ The crystal structure of AtILL2 was published and metal and substrate binding sites have been proposed. ${ }^{[61]}$ Based on the crystal structure of the AtILL2 apoenzyme (without metal cofactor in the metal binding site)
Bitto et al. (2009) ${ }^{[61]}$ suggested a model for auxin conjugate hydrolase activity towards the preferred substrate IAA-Ala. The authors compared their model of the Arabidopsis hydrolase with sequences from other species. Based on the different substrate specificities found, they pointed out several possible amino acid residues in the active center which could be responsible for changes in activity. For example, Leu175 was hypothesized to be responsible for selectivity against IAA-amino acid conjugates with amino acid side chains bulkier than alanine or serine. ${ }^{[61]}$ However, Leu175 is not conserved in Arabidopsis amidohydrolases, it is only present in ILL2 and in its closest homolog ILL1. In ILR1, this residue is replaced by Tyr176, which could stabilize the aromatic side chains of the preferred substrates of this amidohydrolase isoform (IAA-Phe or IAA-Tyr) by a stacking interaction. ${ }^{[61]}$ The wheat IAR3 homolog (TaIAR3) contains Gly168 in the corresponding position of the putative selectivity filter residue Leu175 in AtILL2. The only other notable difference is a single residue insertion (Thr375 of TalAR3) located in the vicinity of the residues forming the hydrophobic cavity for the indole ring. It is possible that these two modifications within the active site of TaIAR3 contribute to the ability of this enzyme to productively bind and hydrolyze auxin derivatives with longer side chains. Based on the same crystal structure, Savić et al. (2009) ${ }^{[42]}$ modeled the BrILL2 protein, which shows strong activity against longer chain auxin amino acid conjugates. While the activity of auxin conjugate hydrolases has been
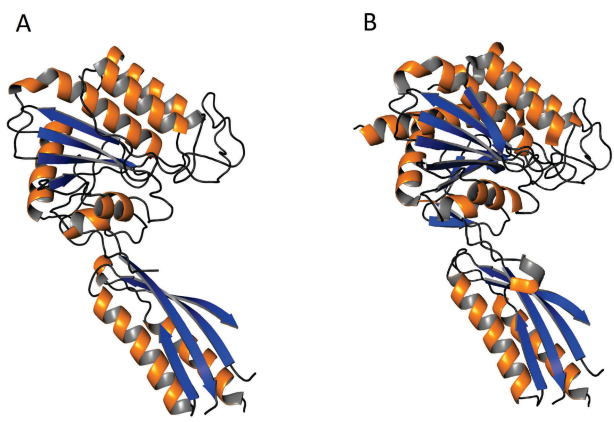

Figure 1. (A) A proposed model of the putative Physcomitrella patens PpIAR3 (UniProt: A9U840) made by Phyre ${ }^{[56]}$ using AtILL2 crystal structure (Protein Data Bank code: $2 \mathrm{Q} 43$ ) as a template (33 \% homology). The putative PpIAR3 protein sequence (XP_001787023.1) ${ }^{[54]}$ has been aligned to $P$. patens protein dataset in Ensembl Plants, ${ }^{[57]}$ with default parameters, to yield a $100 \%$ homologous sequence to the protein product of PP1S9535_1V6.1 transcript. The model was visualized in Pymol; (B) Crystal structure of the AtILL2 (Protein Data Bank code: 2Q43), visualized in Pymol. 


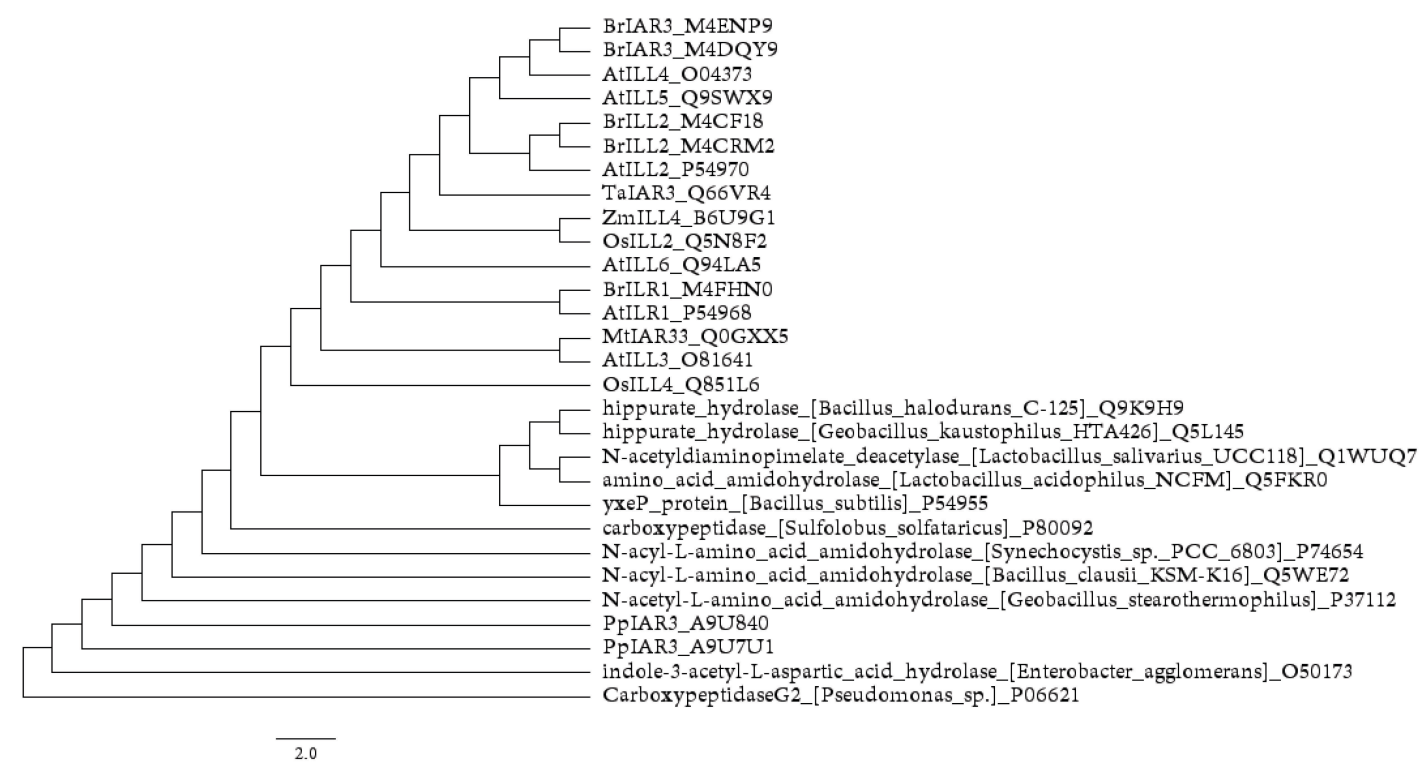

Figure 2. Neighbor-joining phylogram of the M20 peptidase family members orthologous to the A. thaliana auxin amidohydrolases (AAHs). The sequences of plant AAHs and bacterial homologs ${ }^{[62]}$ were retrieved from UniProt (the two-letter prefix before the AAH name denotes the species from which the AAH originates, $\mathrm{Br}$ - from Brassica rapa, At - from A. thaliana, As - from A. suecica, Mt - from Medicago truncatula , Ta - from Triticum aestivum, Pp - from Physcomitrella patens, Os- from Oryza sativa, Zm- from Zea mays, UniProt IDs are given after the last underscore in a respective AAHs taxa/protein names), and were aligned in Jalview2.10.3b1 ${ }^{[63]}$ by Muscle alignment. ${ }^{[64]}$ Phylogram was created by the Jalview in-built neighbor-joining tree calculation using BLOSUM62 parameters on region from MuscleWS alignment. The visualization of the tree was done by FigTree v1.4.3. ${ }^{[65]}$

determined using the native enzymes, site-directed mutagenesis could add biochemical information about important amino acid residues and help to answer the question whether only a few amino acid changes are necessary to convert an enzyme specific for IAA (i.e. the Arabidopsis family) to IBA / IPA amino acid conjugate hydrolases (i.e. Brassica). Also, this approach could improve our understanding of the evolution of auxin amidohydrolases, for example, how the remodeling of the active site happened during evolution, which enabled for the change of preference for zinc to manganese ions in the active site. ${ }^{[61]}$ Based on the sequence alignment of bacterial representatives of the M20 family ${ }^{[62]}$ and the plant amidohydrolases, a few conserved motifs are present. ${ }^{[50,59]}$ Promiscuity of the auxin amidohydrolases to accept structurally different substrates into the active site cavity ${ }^{[41-43]}$ has probably arisen from the changes in the amino acid sequence of the active site cavity and/or in the conserved structural motifs present in all representatives of the M20 family, which lead to a fine tuning of the ability of an ancestor of auxin amidohydrolases to cleave various substrates across different families of land plants. ${ }^{[3]}$ Quite possibly, the ancestor was a peptidase which was able to cleave a structurally related dipeptide, Trp-Asp as a substrate. ${ }^{[3]}$ For example, a recent implication of a putative auxin amidohydrolase PpIAR3 from moss Physcomitrella patens in a phylogenetic study of the evolutionary relationship among hydrolases from both gymnosperms and angiosperms, employed the PpIAR3 as an outgroup, implying several hydrolases from gymnosperms as molecular ancestors of all known hydrolases. ${ }^{[54]}$ The protein sequences of plant auxin amidohydrolases (AAHs) retrieved from UniProt and bacterial homologues belonging to the M20 family of hydrolases ${ }^{[62]}$ were used for phylogenetic tree construction (Figure 2.) The phylogram, wherein the bacterial carboxypeptidase G2 was employed as an outgroup, shows bacterial hydrolases clearly clade separately from angiosperm plant hydrolases, together with the putative $P$. patens hydrolase PpIAR3. This hydrolase therefore is relatively primitive in terms of its evolutionary relationships with other plant AAHs. The presence of ILR1-like orthologues in bacterial species has been suggested as direct evidence of the extremely ancient roots of this gene family, ${ }^{[38]}$ however many prokaryotic species express hydrolases which are members of the M20 peptidase family (illustrated in Figure 2.), and what is considered extremely ancient might actually be the conserved peptidase domains seen in all these various hydrolases. ${ }^{[40]}$ Based on low sequence homology with bacterial hydrolases, plant AAHs may have emerged as a 
result of convergent evolution and not from bacteria. ${ }^{[40]}$ The question arises where does the moss PpIAR3 fit in the story, concerning its substrate specificity and activity towards auxin conjugates, as it shows a higher homology with the bacterial peptidases, than with the hydrolases from higher plants (only $33 \%$ homology with AtILL2) (Figure 2.), but according to homology modeling remains in the structural fold typical of all the hydrolases from the M20 family (Figure 1.A). New insights into the structure- function relationship of the putative PpIAR3 would add to our understanding of the evolution of all auxin amidohydrolases.

So far, modeling and subsequent analysis of mutant enzymes has been carried out for a bacterial auxin conjugate hydrolase specific for IAA-Asp, ${ }^{[50]}$ and now a few studies on plant enzyme counterparts have been added to the picture, ${ }^{[42,58]}$ following the publication of the crystal structure of Arabidopsis ILL2 (an IAA specific auxin conjugate hydrolase, Fig. 1. B), ${ }^{[61]}$ which is available and has been used for modeling studies. ${ }^{[42,58,61]}$

All auxin amidohydrolases possess $\mathrm{N}$ - and $\mathrm{C}$-terminal protein sequences suggesting their localization in endoplasmic reticulum (ER). Recent study proved that IAR3, ILL2 and ILR1 of $A$. thaliana reside in the ER, indicating that in this compartment the hydrolases regulate the rates of amido-IAA hydrolysis, which results in activation of auxin signalling. ${ }^{[66]}$

\section{Potential Role in Plant Growth, Development and Stress Responses}

The role of auxin conjugate hydrolases, however, is far from being clear. The Arabidopsis mutants with a defect in one hydrolase gene do not show any aberrant phenotype besides lower sensitivity on the IAA conjugate that the respective enzyme is able to cleave. ${ }^{[51]}$ Only a triple mutant combining mutations in the three most abundant hydrolases showed phenotypes related to root and shoot growth. ${ }^{[50]}$ Several of these auxin conjugate hydrolases seem to play a role during symbiosis of $M$. truncatula with Sinorhizobium and arbuscular mycorrhiza because specific transcripts were upregulated during early stages of the interaction. ${ }^{[43]}$

Several publications reported an involvement of several members of auxin-amidohydrolases in abiotic stress responses. Thus, an Arabidopsis ILL3-homolog from Populus was isolated during salt stress experiments, implicating a role for free IAA in stress situations. [67] Overexpression of the poplar auxin conjugate hydrolase rendered Arabidopsis more tolerant to salt stress than wild type. Proteome analysis of soybean roots at an early vegetative stage identified an auxin-amidohydrolase, which was induced upon waterlogging stress, suggesting that the release of free auxin is necessary for the adventitious root formation as an adaptation to the flooded conditions. ${ }^{[68]}$
The authors suggested that activation of IAR3 contributed to free auxin that mediates root architecture under water limitation. Furthermore, it was shown that the level of the IAR3 transcript was increased in Arabidopsis due to high osmotic stress.[69]

Also, the fact that some of the auxin conjugate hydrolases isolated so far prefer IBA- conjugates over IAA conjugates, for example wheat ${ }^{[41]}$ and Chinese cabbage ${ }^{[42]}$ has yet been neglected. The much higher activity of several auxin conjugate hydrolases with IBA and IPA could be an indication that these enzymes play different roles for the respective plant species. In addition, AtIAR3 was shown to hydrolyze amino acid conjugates with JA in addition to IAA conjugates, indicating a possible role in plant defense or wound response. ${ }^{[70,71]}$ Since then, further evidence for the hydrolysis of JA conjugates has been presented. ${ }^{[52,53]} \mathrm{A}$ recent report showed that expression of three auxinamidohydrolase genes, ILR1, ILL6, and IAR3, was upregulated in leaves by wounding or JA treatments. ${ }^{\text {[2] }}$ Furthermore, it was also found that JA treatment induced the IAR3 transcript level and consequently IAA level in $B$. rapa seedlings. ${ }^{[73]}$

Besides abiotic stress, auxin amidohydrolases were shown to be involved in biotic stress responses. Work from $B$. rapa indicates the involvement of hydrolases in the interaction of the host plant with the obligate biotrophic root pathogen Plasmodiophora brassicae. ${ }^{[60]}$ Also, the work on the response of Solanaceae family plants, Nicotiana benthamiana, tomato and potato, to biotrophic and hemibiotrophic pathogen attack indicated the role of IAR3 orthologs in the alternations of auxin homeostasis that consequentially lead to changes in plant defense responses. ${ }^{\text {[74] }}$ Knocking-down the expression of IAR3 genes by virus-induced gene silencing in $N$. benthamiana and tomato plants caused lowering of free IAA levels and modified the susceptible plants' responses to the biotic stressor by enhancing basal defenses and improving resistance to infection.

The most likely function of the ILR1 / IAR3-like gene family in dicots and monocots is to provide free IAA from conjugated auxin when levels of the hormone begin to decrease, but the answer may be more complicated. Davies et al. (1999),, ${ }^{[37]}$ Campanella et al. (2003),, ${ }^{[18]}$ and Rampey et al. (2004) ${ }^{[51]}$ have examined basic ILR1, IAR3, and sILR1 temporal and spatial expression, but a more detailed understanding is needed of how these enzymes regulate auxin levels and are themselves regulated. Question arises why, for example, IAR3, ILR1, and sILR1 are all most highly expressed in the roots? ${ }^{[18,37,51]}$ Is this high root expression common for other members of the ILR1-like ortholog family? What role do the IBA conjugates and IBA amidohydrolases play? These observations and unanswered questions reveal that this family of enzymes has complexities that have not yet been examined. 


\section{CONCLUSION}

Auxin amidohydrolases are metalloenzymes involved in homeostasis of the plant hormone auxin. They hydrolyze auxin amidoconjugates releasing free active auxin on the plant's demand. There are a number of auxin amidohydrolase homologs with overlapping substrate specificities found in many species. It was reported so far that auxin amidohydrolases are involved in diverse processes of plant growth and development, symbiotic relationship of plants and arbuscular mycorrhiza, as well as plant stress responses (abiotic and biotic). However, there are numerous unanswered questions on their role and regulation that need to be addressed in future research. A combination of functional research comprising auxin amidohydrolases mutants and overexpressors with traditional biochemical studies will need to provide a more detailed view for understanding the role and regulation of these enzymes in plant growth and development as well as plant survival in, often, unfriendly environment.

Acknowledgment. This work was supported by the Croatian Science Foundation (project no. IP-2014-09-4359).

\section{REFERENCES}

[1] N. D. Rawlings, M. Waller, A. J. Barrett, A. Bateman, Nucleic Acids Res. 2014, 42, D503.

[2] C. M. Seibert, F. M. Raushel, J. Biochem. 2005, 44, 6383.

[3] J. Ludwig-Müller, J. Exp. Bot. 2011, 62, 1757.

[4] C. S. Westfall, A. M. Muehler, J. M. Jez, J. Biol. Chem. 2013, 288, 19304.

[5] B. Kojić-Prodić, V. Magnus, Annals. The European Academy of Sciences 2005, (1379-1982), 310.

[6] B. Salopek-Sondi, I. Pavlović, A. Smolko, D. Šamec in Mechanism of Plant Hormone Signaling Under Stress (Ed: G. Pandey), John Wiley \& Sons Inc., New Jersey, 2017, pp. 3-36.

[7] B. Kojić-Prodić , B. Nigović, S. Tomić, N. Ilić, V. Magnus, R. Konjević, Z. Giba, W.L. Duax, Acta Crystallogr., Sect. B: Struct. Sci. 1991, 1010.

[8] S. Antolić, B. Kojić-Prodić, S. Tomić, B. Nigović, V. Magnus, J. D. Cohen, Acta Crystallogr. B52 1996, 651.

[9] S. Antolić, B. Salopek, B. Kojić-Prodić, V. Magnus, J. D. Cohen, Plant Growth Regul. 1999, 27, 21.

[10] V. Magnus, B. Kojić-Prodić, Phyton 1999, 39, 9.

[11] B. Nigović, B. Kojić-Prodić, S. Antolić, S. Tomić, V. Puntarec, J.D. Cohen, Acta Crystallogr. B52 1996, 332.

[12] B. Nigović, S. Antolić, B. Kojić-Prodić, R. Kiralj, V. Magnus, B. Salopek-Sondi, Acta Crystallogr. B, Struct. Sci. 56 2000, 1, 94.
[13] Q. Ma, P. Grones, S. Robert, J. Exp. Bot. 2018, 69, 313.

[14] K. Ljung, A.K. Hull, M. Kowalczyk, A. Marchant, J. Celenza, J.D. Cohen, G. Sandberg, Plant Mol. Biol. 2002, 49, 249.

[15] C. Seidel, A. Walz, S. Park, J.D. Cohen, J. LudwigMüller, Plant Biol. 2006, 8, 340.

[16] J. D. Cohen, R. S. Bandurski, Annu. Rev. Plant Physiol. 1982, 33, 403.

[17] R. S. Bandurski, J. D. Cohen, J. P. Slovin, D. M. Reinecke in: Plant Hormones: Physiology, Biochemistry and Molecular Biology, 2nd edition ( $\mathrm{Ed}$ : P. Davies), Kluwer Academic Publishers, Boston, 1995, pp. 39-65.

[18] J. J. Campanella, J. Ludwig-Müller, V. Bakllamaja, V. Sharma, A. Cartier, Plant Growth Regul. 2003, 41, 215.

[19] R. P. Hangarter, N. E. Good, Plant Physiol. 1981, 68, 1424.

[20] J. Lasswell, L.E. Rogg, D.C. Nelson, C. Rongey, B. Bartel, B. Plant Cell. 2000, 12, 2395.

[21] A. Bajguz, A. Piotrowska, Phytochemistry 2009, 70, 957.

[22] J. M. Sonner, W. K. Purves, Plant Physiol. 1985, 77, 784.

[23] J. D. Cohen, Plant Physiol. 1982, 70, 749.

[24] A. Östin, T. Moritz, G. Sandberg, Biol. Mass Spectrum. 1992, 21, 292.

[25] N. M. Barratt, W. Dong, D. A. Gage, V. Magnus, C. D. Town, Physiol. Plant. 1999, 105, 207.

[26] Y. Y. Tam, J. Normanly, Physiol Plant 2002, 115, 513.

[27] M. Kowalczyk, G. Sandberg, Plant Physiol. 2001, 127, 1845.

[28] A. Pencik, J. Rolcik, O. Novák, V. Magnus, P. Bartak, R. Buchtik, B. Salopek-Sondi, M. Strnad, Talanta 2009, 80, 651.

[29] O. Novák, E. Hényková, I. Sairanen, M. Kowalczyk, T. Pospíšil, K. Ljung, Plant J. 2012, 72, 523.

[30] A. Walz, S. Park, J. P. Slovin, J. Ludwig-Müller, Y. S. Momonoki, J. D. Cohen, Proc. Natl. Acad. Sci. U. S. A. 2002, 99, 1718.

[31] A. Walz, C. Seidel, G. Rusak, S. Park, J. D. Cohen, J. Ludwig-Müller, Planta 2008, 227, 1047.

[32] S. Park, J. D. Cohen, J. P. Slovin, Planta 2006, 224, 1015.

[33] S. Park, J. A. Ozga, J. D. Cohen, D. M. Reinecke, J. Plant Growth Regul. 2010, 28, 184.

[34] P. E. Staswick, I.Tiryaki, M. L. Rowe, Plant Cell 2002, 14, 1405.

[35] P. E. Staswick, B. Serban, M. Rowe, I. Tiryaki, M. T. Maldonado, M. C. Maldonado, W. Suza, Plant Cell 2005, 17, 616.

[36] B. Bartel, G. Fink, Science 1995, 268, 1745 
[37] R. Davies, D. Goetz, J. Lasswell, M. Anderson, B. Bartel, Plant Cell 1999, 11, 365.

[38] S. LeClere, R. Tellez, R. A. Rampey, S. P. T. Matsuda, B. Bartel., J. Biol. Chem. 2002, 277, 20446.

[39] J. J. Campanella, V. Bakllamaja, T. Restieri, M. Vomacka, J. Herron, M. Patterson, S. Shahtaheri, Plant Growth Regul. 2003, 39, 175.

[40] J. J. Campanella, D. Larko, J. Smalley, Comp. Funct. Genomics 2003, 4, 584.

[41] J. J. Campanella, A. Olajide, V. Magnus, J. LudwigMüller, Plant Physiol. 2004, 135, 2230.

[42] B. Savić, S. Tomić, V. Magnus, K. Gruden, K. Barle, R. Grenković, J. Ludwig-Müller, B. Salopek-Sondi, Plant Cell Physiol. 2009, 50, 1587.

[43] J. J. Campanella, S. M. Smith, D. Leibu, S. Wexler, J. Ludwig-Müller, J. Plant Growth Regul. 2008, 27, 26.

[44] J. Ludwig-Müller, Plant Growth Regul. 2000, 32, 219.

[45] J. Ludwig-Müller, J.D. Cohen, Physiol. Plant. 2002, 115, 320.

[46] E. M. Frick, L. C. Strader, J. Exp. Bot. 2018, 69, 169.

[47] J. Hagemeier, B. Schneider, N. J. Oldham, K. Hahlbrock, Proc. Natl. Acad. Sci. USA 2001, 98, 753.

[48] L. M. Segal, F. Wightman, Physiol. Plant. 1982, 56, 367.

[49] E. A. Schneider, C. W. Kazakoff, F. Wightman, Planta 1985, 165, 232.

[50] J.-C. Chou, W. W. Mulbry, J. D. Cohen, Mol. Gen. Genet 1998, 259, 172.

[51] R. A. Rampey, S. LeClere, M. Kowalczyk, K. Ljung, G. Sandberg, B. Bartel, Plant Physiol.2004, 135, 978.

[52] M. G. Woldemariam, N. Onkokesung, I. T. Baldwin, I. Galis, Plant J. 2012, 72, 758.

[53] E. Widemann, L. Miesch, R. Lugan, E. Holder, C. Heinrich, Y. Aubert, M. Miesch, F. Pinot, T. Heitz, J. Biol. Chem. 2013, 288, 31701.

[54] J. J. Campanella, N. Zaben, D. Enriquez, J. V. Smalley, J. Ludwig-Müller, Acta Hort. 2014, 1042, 79.

[55] B. Andersson, G. Sandberg, J. Chromatogr. 1982, 238, 151.

[56] L. A. Kelley, M. J. E. Sternberg, Nat. Protoc. 2009, 4, 363.

[57] EsemblPlants, Blast search, can be found under https://plants.ensembl.org/Physcomitrella_patens/ Tools/Blast?db=core.
[58] A. Smolko, F. Šupljika, J. Martinčić, N. Jajčanin-Jozić, M. Grabar-Branilović, S. Tomić, J. Ludwig- Müller, I. Piantanida, B. Salopek-Sondi, Phys. Chem. Chem. Phys. 2016, 13, 8890.

[59] D. S. Auld in: Handbook of Proteolytic Enzymes (Eds: N.D. Rawlings, G. Salvesen), Academic Press, 2013, pp. 370-396.

[60] A. Schuller, J. Ludwig-Müller, New Phytol. 2006, 171, 145.

[61] E. Bitto, C. A. Bingman, L. Bittova, N. L. Houston, R. S. Boston, B. G. Fox, G. N. Phillips Jr., Proteins 2009, $74,61$.

[62] J.-C. Chou, W. H. Welch, J. D. Cohen, Plant Cell Physiol. 2004, 45, 1335.

[63] A. M. Waterhouse, J. B. Procter, D. M. A Martin, M. Clamp, G. J. Barton, Bioinformatics 2009, 25, 1189.

[64] R. C. Edgar, Nucleic Acids Res. 2004, 32, 1792.

[65] Molecular evolution, phylogenetics and epidemiology, software, can be found under tree.bio.ed.ac.uk.

[66] A. P. Sanchez Carranza, A. Singh, K. Steinberger, K. Panigrahi, K.Palme, A. Dovzhenko, C. Dal Bosco, Sci. Rep. 2016, 6, 24212.

[67] U. Junghans, A. Polle, P. Düchtig, E. Weiler, B. Kuhlman, F. Gruber, T. Teichmann, Plant Cell Environ. 2006, 29, 1519.

[68] I. Alam, D. G. Lee, K. H. Kim, C. H. Park, S. A. Sharmin, H. Lee, J. Biosci. 2010, 35, 49.

[69] N. Kinoshita, H. Wang, H. Kasahara, J. Liu, C. MacPherson, Y. Machida, Y. Kamiya, M.A. Hannah, N. H. Chua, Plant Cell 2012, 24, 3590.

[70] B. Bartel, S. LeClere, M. Magidin, B.K. Zolman, J. Plant Growth Regul. 2001, 20, 198.

[71] E. Titarenko, E. Rojo, J. Leon, J. J. Sanchez-Serrano, Plant Physiol. 1997, 115, 817.

[72] T. Zhang, A. N. Poudel, J. B. Jewell, N. Kitaoka, P. Staswick, H. Matsuura, A. J. Koo, J. Exp. Bot. 2016, 67, 2107.

[73] B. Salopek-Sondi, D. Šamec, S. Mihaljević, A. Smolko, I. Pavlović, I. Janković, J. Ludwig-Müller, Plant Cell Rep. 2013, 32, 1031.

[74] S. D'Ippolito, R. Vankova, M. H. Joosten, C. A. Casalongué, D. F. Fiol, Plant Sci. 2016, 253, 31. 